\title{
Ultrasound guided electrochemotherapy for the treatment of a clear cell thymoma in a cat
}

\author{
Enrico Pierluigi Spugnini ${ }^{1, *}$, Francesco Menicagli ${ }^{2}$, Michela Pettorali ${ }^{2}$ and Alfonso Baldi ${ }^{1,3}$ \\ ${ }^{1}$ Biopulse s.r.l., via Toledo 256, 80132 Naples, Italy \\ ${ }^{2}$ Gianicolense Veterinary Centre, Via Lorenzo Valla 25/b, 00152 Rome, Italy \\ ${ }^{3}$ Department of Environmental, Biological and Pharmaceutical Sciences and Technologies, Università della \\ Campania "Luigi Vanvitelli", Caserta, Italy
}

\begin{abstract}
A twelve-year-old male castrated domestic shorthair cat was presented for rapidly progressing respiratory distress. The cat was depressed, tachypneic and moderately responsive. Ultrasonography showed a mediastinal mass associated with a significant pleural effusion that needed tapping every five to seven days. Ultrasound guided biopsy yielded a diagnosis of clear cell thymoma upon histopathology. After complete staging procedures, the owner elected to treat the cat with electrochemotherapy (ECT) using systemic bleomycin. Two sessions of ultrasound guided ECT were performed at two week intervals with trains of biphasic electric pulses applied using needle electrodes until complete coverage of the area was achieved. The treatment was well tolerated and resulted in partial remission (PR). Additional sessions were performed on a monthly basis. The cat is still in PR after fourteen months. ECT resulted in improved local control and should be considered among the available adjuvant treatments in pets carrying visceral tumors.
\end{abstract}

Keywords: Biphasic pulses, Bleomycin, Electrochemotherapy, Feline, Thymoma.

\section{Introduction}

Thymomas are uncommon neoplasms that are typically located in the cranioventral mediastinum in domestic species, which generally affects older animals (Biller and Larson, 2010). The clinical presentation of these tumors can be directly influenced by the neoplasm, consisting with respiratory distress, or could be characterized by paraneoplastic syndromes such as myasthenia gravis (rare in cats compared to dogs) (Singh et al., 2010; Shilo et al., 2011; Hague et al., 2015) or exfoliative dermatitis, prevalently reported in cats (Rottenberg et al., 2004; Singh et al., 2010). Treatment options in cats involve surgical excision (Gores et al., 1994; Zitz et al., 2008), with reported median survival times for patients surviving the surgery of 1,825 days, with a 1-year survival rate of $89 \%$ and a 3-year survival rate of 74\% (Zitz et al., 2008) or radiation therapy with a median survival time of 720 days (Smith et al., 2001). In this article we report the treatment of a clear cell thymoma in a cat by means of ultrasound guided electrochemotherapy.

\section{Case details}

A twelve-year-old male castrated domestic shorthair cat was presented for rapidly progressing respiratory distress (ten days). Upon physical examination, the patient was depressed, tachypneic and moderately responsive. Staging procedure included complete blood cell count, biochemical analysis and urinalysis (all the tests were within normal limits). Additionally, thoracic and abdominal ultrasonographic exams were performed. Thoracic ultrasonography showed a large mediastinal mass consisting of a solid neoformation with cystic areas, having heterogeneous echogenicity with a major diameter of $7.5 \mathrm{~cm}$. Moreover the mass was associated with compression of the adjacent organs and a significant pleural effusion $(200 \mathrm{ml})$ that needed repeated tapping every five to seven days (Fig. 1A). Differential diagnoses included mediastinal lymphoma, thymoma, ectopic thyroid carcinoma and chemodectoma. The features of the mass, accordingly to current literature, were suggestive of a thymoma (Patterson and Marolf, 2014). Following removal of the thoracic effusion, ultrasonography guided biopsy was performed, under sedation with tramadol (Altadol, Formevet, Milan, Italy) and medetomidine (Domitor, Pfizer Italia, Milan, Italy) SC injection at the dose of 3 $\mathrm{mg} / \mathrm{kg}$ and $40 \mu \mathrm{g} / \mathrm{kg}$, respectively, followed by propofol (Rapinovet, Intervet Italia, Milan, Italy) at the dosage of $2 \mathrm{mg} / \mathrm{kg}$. Biopsy was performed collecting four tissue samples from different areas of the mass by using an automated 16 gauge tru-cut gun (Biopince, Argon Medical Devices, Texas, USA). The cat did not experience any side effects from the procedure. The histopathology report came back with a diagnosis of clear cell thymoma. This is an unusual variant characterized by large round cells with abundant clear cytoplasm and distinct cytoplasmic margins (Fig. 1B) (Mackey, 1975; Mettler and Hauser, 1984). 

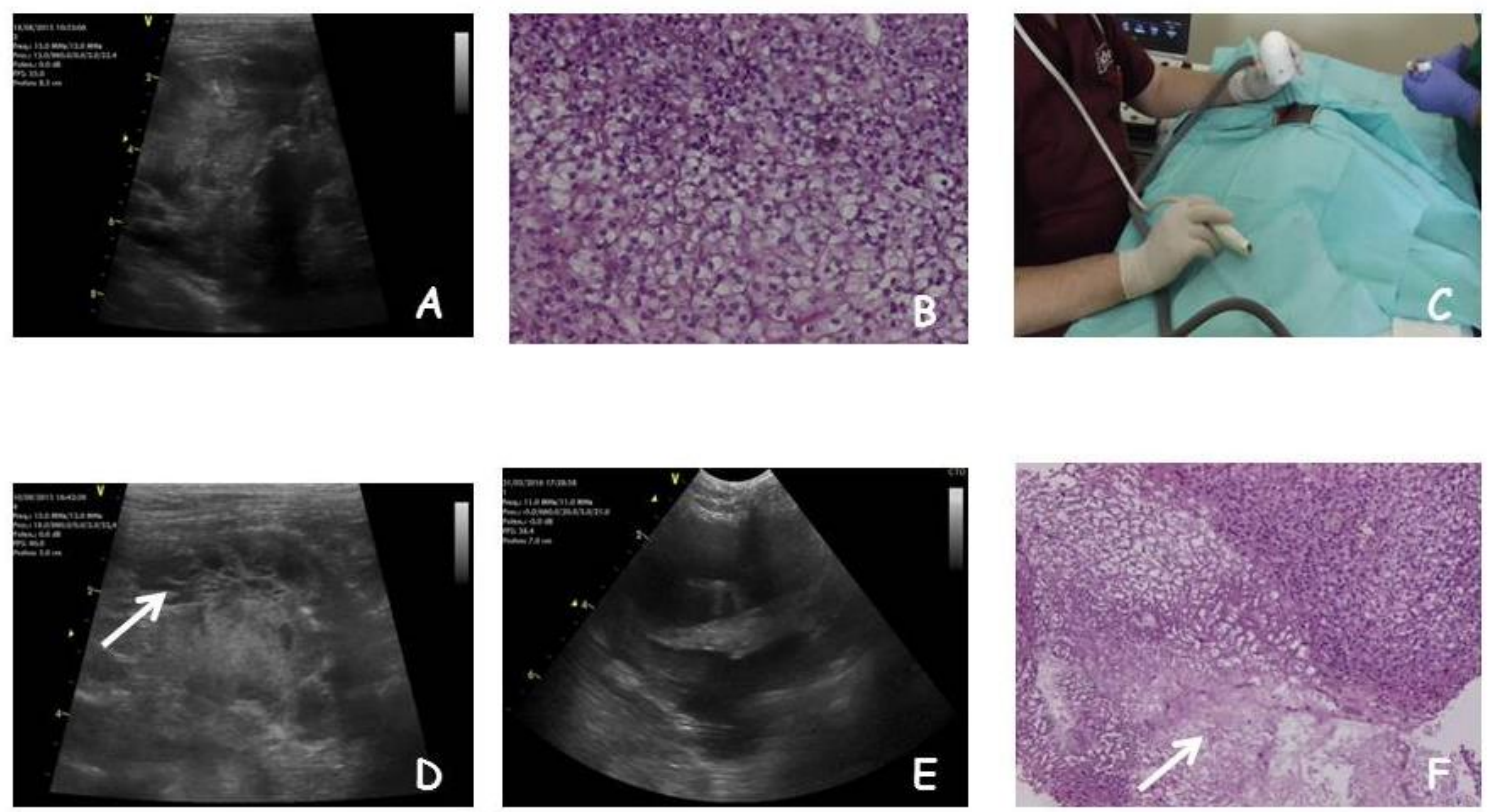

Fig. 1. (A): Ultrasonographic appearance of the thymoma at presentation. (B): Histopathological features of the clear cell thymoma showing the characteristic large round cells with abundant clear cytoplasm (H\&E, original magnification x 10). (C): An ultrasound guided ECT session. (D): The ultrasonographic appearance of the needle array (arrow). (E): The tumor after two sessions of ECT, note the replacement of solid areas by cystic formations. (F): The histopathological appearance of the thymoma after ECT, showing partial replacement of neoplastic tissue by necrotic and fibrotic tissue (arrow) (H\&E, original magnification x 5).

Standard therapies including surgery and radiation therapy were offered to the owner, who declined for emotional and economic reasons. The owner elected the cat to be treated with systemic chemotherapy and the patient was treated with a multidrug protocol including prednisone $1 \mathrm{mg} / \mathrm{kg} \mathrm{BID}$, vincristine $(0.7$ $\mathrm{mg} / \mathrm{m}^{2}$ IV on week one), chlorambucil $(1.4 \mathrm{mg} / \mathrm{kg}$ PO on week two), and doxorubicin $\left(20 \mathrm{mg} / \mathrm{m}^{2} \mathrm{IV}\right.$ on week three), unfortunately without significant improvement after one cycle of each drug.

During this period the cat needed two additional tappings at week two and four of the multi-drug protocol. Thoracic ultrasonographic exam was performed at weeks two and four of the multi-drug protocol. At this point, (specifically seven weeks from the initial diagnosis) the owner elected the cat to be treated with ultrasound guided ECT. A complete staging process was performed as above described. The hematological and biochemical exams were within normal limits. The cat was sedated as above described and bleomycin (Bleomicina Solfato, Sanofi-aventis, Milan, Italy) at the dose of $20 \mathrm{mg} / \mathrm{m}^{2}$ was administered as an i.v. bolus as elsewhere described (Spugnini et al., 2015).

Ultrasonographic measure of the tumor was performed, leading to an observed major diameter of $7 \mathrm{~cm}$. Five minutes after the injection, five trains of 8 biphasic pulses at the voltage of $800 \mathrm{~V} / \mathrm{cm}, 1 \mathrm{~Hz}$ frequency, lasting $50+50 \mu \mathrm{s}$ with $300 \mu \mathrm{s}$ interpulse (total treatment time per train $3.2 \mathrm{~ms}$ ) were administered by means of a clinical electroporator certified for veterinary application (Onkodisruptor ${ }^{\circledR}$, Biopulse S.r.l., Naples, Italy) using a specially paired needle array electrodes (Patent application EP2221086A1) until complete coverage of the lesion was achieved (Fig. 1C). This electrode has a specially devised nonconductive shielding that leaves as active parts the final $1.5 \mathrm{~cm}$ of the needles and is ultrasonographically detectable (Fig. 1D). The high number of pulses was delivered to increase the chance of local control through the combination of the direct tissue ablation induced by the Joule effect of the heating electrodes and the increased drug uptake induced by ECT (Spugnini et al., 2016).

The patient did not show any side effects (as confirmed by pulse oximetry, electrocardiography and imaging studies) due to the therapy and was discharged under antibiotics and non-steroidal anti-inflammatory drugs: enrofloxacin $5 \mathrm{mg} / \mathrm{kg}$ PO SID (Baytril, Bayer, Milan, Italy) and meloxicam at the dose of $0.05 \mathrm{mg} / \mathrm{kg}$ PO SID (Metacam, Boehringer Ingelheim, Milan, Italy) for six days. A complete blood cell count and biochemical analysis were performed one week after ECT. A thoracic ultrasonograpic exam and second ECT session were performed two weeks later. At this time the mass was stable and there was no effusion.

After two additional weeks ultrasonography evidenced a 50\% tumor reduction (major diameter $3.5 \mathrm{~cm}$ ) with the formation of larger cystic areas (Fig. 1E). Tumor biopsies of the solid areas were performed at this time 
to confirm tumor response. Histopathology exam evidenced the replacing of large areas of tumor by necrotic and sclerotic tissue, consistent with response to ECT (Fig. 1F) (Spugnini et al., 2007).

The cat was treated with a maintenance ECT protocol consisting with a monthly session until complete response or progressive disease was observed. Response was assessed through monthly ultrasonographic exam and one week after ECT a complete blood cell count and biochemical profile were performed to monitor for side effects.

Toxicoses were not detected during rechecks. The cat is still in PR after fourteen months from the beginning of ECT, the mass is reduced to a stable approximate diameter of $1.5 \mathrm{~cm}$ and the only therapy is a tapping when needed. Overall the cat is having a good quality of life.

\section{Discussion}

ECT is a therapy aimed at increasing the loco-regional control of solid tumors that is being included among the standard oncology therapies thanks to its low cost and minimal side effects combined with a significant efficacy (Spugnini, 2016). This technique is based on the delivery of permeabilizing electric pulses on tumors or tumors' beds after the local or systemic administration of a lipophobic chemotherapy agent (Spugnini and Baldi, 2014).

In feline medicine, this therapy is currently being added to the first lines of treatment for soft tissue sarcoma and carcinoma (Spugnini et al., 2011, 2015; Spugnini, 2016). Treatments for feline thymoma include surgery and/or radiation therapy. Unfortunately, current veterinary literature is still limited to a few small cohorts of patients (Smith et al., 2001; Zitz et al., 2008). Surgery seems to carry the better prognosis of the two options but may not be always practical, for example in the case of an invasive thymoma. On the basis of emotional factors, ECT was chosen considering its remarkable effectiveness against solid tumors in cats (i.e. fibrosarcoma, squamous cell carcinoma, nasal lymphoma) (Spugnini, 2016).

This is the first report of ultrasound guided ECT in veterinary medicine for mediastinal neoplasms. Despite the close proximity of the other thoracic organs, ECT has been well tolerated and after more than one year since the beginning, the cat is still experiencing a durable PR. To the best of our knowledge this is the first report of clear cell thymoma in a cat treated with ECT. Further investigations are warranted to confirm this preliminary finding and to evaluate the effectiveness of ultrasound guided ECT for the treatment of visceral tumors in companion animals.

\section{Conflict of Interest}

Enrico P.Spugnini and Alfonso Baldi are stockholders of Biopulse S.r.l.

\section{References}

Biller, D.S. and Larson, M.M. 2010. Mediastinal disease. In: Textbook of veterinary internal medicine, Eds., Ettinger S. J., Feldman, E.C. St. Louis, MO: Saunders, pp: 1119-1124.

Gores, B.R., Berg, J., Carpenter, J.L. and Aronsohn, M.G. 1994. Surgical treatment of thymoma in cats: 12 cases (1987-1992). J. Am. Vet. Med. Assoc. 204, 1782-1785.

Hague, D.W., Humphries, H.D., Mitchell, M.A. and Shelton, G.D. 2015. Risk Factors and Outcomes in Cats with Acquired Myasthenia Gravis (20012012). J. Vet. Intern. Med. 29, 1307-1312.

Mackey, L. 1975. Clear-cell thymoma and thymic hyperplasia in a cat. J. Comp. Pathol. 85, 367-371.

Mettler, F. and Hauser, B. 1984. Clear cell thymoma in a dog. J. Comp. Pathol. 94, 315-317.

Patterson, M.M. and Marolf, A.J. 2014. Sonographic characteristics of thymoma compared with mediastinal lymphoma. J. Am. Anim. Hosp. Assoc. 50, 409-413.

Rottenberg, S., von Tscharner, C. and Roosje, P.J. 2004. Thymoma-associated exfoliative dermatitis in cats. Vet. Pathol. 41, 429-433.

Shilo, Y., Pypendop, B.H., Barter, L.S. and Epstein, S.E. 2011. Thymoma removal in a cat with acquired myasthenia gravis: a case report and literature review of anesthetic techniques. Vet. Anaesth. Analg. 38, 603-613.

Singh, A., Boston, S.E. and Poma, R. 2010. Thymomaassociated exfoliative dermatitis with postthymectomy myasthenia gravis in a cat. Can. Vet. J. $51,757-760$.

Smith, A.N., Wright, J.C., Brawner, W.R. Jr, LaRue, S.M., Fineman, L., Hogge, G.S., Kitchell, B.E., Hohenhaus, A.E., Burk, R.L., Dhaliwal, R.S. and Duda, L.E. 2001. Radiation therapy in the treatment of canine and feline thymomas: a retrospective study (1985-1999). J. Am. Anim. Hosp. Assoc. 37, 489-496.

Spugnini, E.P., Baldi, F., Mellone, P., Feroce, F., D'Avino, A., Bonetto, F., Vincenzi, B., Citro, G. and Baldi, A. 2007. Patterns of tumor response in canine and feline cancer patients treated with electrochemotherapy: preclinical data for the standardization of this treatment in pets and humans. J. Transl. Med. 5, 48.

Spugnini, E.P., Renaud, S.M., Buglioni, S., Carocci, F., Dragonetti, E., Murace, R., Cardelli, P., Vincenzi, B., Baldi, A. and Citro, G. 2011. Electrochemotherapy with cisplatin enhances local control after surgical ablation of fibrosarcoma in cats: an approach to improve the therapeutic index of highly toxic chemotherapy drugs. J. Transl. Med. 9, 152. 
Spugnini, E.P. and Baldi, A. 2014. Electrochemotherapy in veterinary oncology: from rescue to first line therapy. Methods Mol. Biol. 1121, 247-256.

Spugnini, E.P., Pizzuto, M., Filipponi, M., Romani, L., Vincenzi, B., Menicagli, F., Lanza, A., De Girolamo, R., Lomonaco, R., Fanciulli, M., Spriano, G. and Baldi, A. 2015. Electroporation Enhances Bleomycin Efficacy in Cats with Periocular Carcinoma and Advanced Squamous Cell Carcinoma of the Head. J. Vet. Intern. Med. 29, 1368-1375.

Spugnini, E.P., Fais, S., Azzarito, T. and Baldi,
A. 2017. Novel Instruments for the Implementation of Electrochemotherapy Protocols: From Bench Side to Veterinary Clinic. J. Cell. Physiol. 232, 490495.

Spugnini, E.P. 2016. Electrochemotherapy in feline oncology. In: August's consultations in feline internal medicine. Ed. Little S.E. Saint Louis, MO, Elsevier publisher, pp: 572-577.

Zitz, J.C., Birchard, S.J., Couto, G.C., Samii, V.F., Weisbrode, S.E. and Young, G.S. 2008. Results of excision of thymoma in cats and dogs: 20 cases (1984-2005). J. Am. Vet. Med. Assoc. 232, 11861192. 